Arts

et Savoirs

\section{Arts et Savoirs}

$2 \mid 2012$

Les théories de l'énonciation : Benveniste après un demi-siècle

\title{
Genre débat d'idées et constructions énonciatives
}

Une réflexion théorico-empirique

Helena T. Valentim et Rosalice Pinto

\section{(c) OpenEdition}

\section{Journals}

Édition électronique

URL : http://journals.openedition.org/aes/504

DOI : $10.4000 /$ aes.504

ISSN : 2258-093X

Éditeur

Laboratoire LISAA

Référence électronique

Helena T. Valentim et Rosalice Pinto, "Genre débat d'idées et constructions énonciatives », Arts et Savoirs [En ligne], 2 | 2012, mis en ligne le 15 juillet 2012, consulté le 01 mai 2019. URL : http:// journals.openedition.org/aes/504; DOI : 10.4000/aes.504

Ce document a été généré automatiquement le 1 mai 2019.

Centre de recherche LISAA (Littératures SAvoirs et Arts) 


\section{Genre débat d'idées et constructions énonciatives}

Une réflexion théorico-empirique

Helena T. Valentim et Rosalice Pinto

\section{Introduction}

1 La problématique de l'énonciation a été mise en évidence par des théoriciens appartenant à plusieurs champs théoriques. D'une part, à partir des études littéraires, Voloschinov (1977) considère que l'énonciation est un produit de l'interaction sociale. Ainsi, elle doit être reliée soit à l'acte de parole inséré dans une situation déterminée, soit à un contexte plus étendu où les aspects historiques doivent être analysés. D'autre part, selon une perspective linguistique, les spécialistes en sciences du langage la conçoivent d'une façon « restreinte » ou « étendue ». Dans le premier cas, Benveniste (1966, 1974), Culioli (1999b), Ducrot (1984), Kerbrat-Orecchioni (1980), par exemple, selon des approches théoriques distinctes, concentrent leur réflexion sur les traces linguistiques présentes dans l'énoncé de l'énonciateur/du locuteur-scripteur et limitent son étude aux aspects essentiellement linguistiques. En revanche, Maingueneau (1986), dans une perspective d'analyse du discours, considère que l'énonciation est le pivot de la relation entre la langue et le monde. De cette façon, elle ne repose pas sur le seul énonciateur, mais elle est construite pendant l'interaction et subit les contraintes multiples des genres discursifs dans lesquels elle s'intègre. Ou encore, pour Bronckart, suivant l'approche de l'interactionnisme sociodiscursif (dorénavant ISD), les mécanismes de responsabilité énonciative semblent être dépendants tantôt des activités sociales et des genres textuels, tantôt des opérations psycho-cognitives qui y sont associées - Bronckart $(2008,89)$. Dans une telle approche, les instances qui assument la responsabilité de l'énonciation ne se présentent plus seulement comme des locuteurs ou des énonciateurs, mais comme des agents, des acteurs ou des actants socialement spécifiés ${ }^{1}$. 
2 En nous inspirant de la perspective textuelle de l'ISD et de la théorie des opérations prédicatives et énonciatives (dorénavant TOPE) développée par Culioli et par ses successeurs, cette contribution portera, d'une part, sur l'identification et la description des procédés énonciatifs - constructions linguistiques - présents dans un genre académique spécifique: le débat d'idées; d'autre part sur les effets contraignants des facteurs génériques sur ces marques formelles linguistiques. Notre objectif, méthodologiquement défiant, est d'essayer de montrer que le croisement et la complémentarité de ces deux cadres théoriques apparemment bien différents peuvent contribuer à enrichir la description de notre corpus. Pour atteindre ces buts, notre argumentation se développera en quatre temps. D'abord, nous présenterons quelques aspects théoriques importants de l'ISD qui serviront d'appui pour nos analyses. Nous évoquerons, ensuite, quelques caractéristiques prototypiques du genre textuel débat d'idées. Ensuite, tout en plaidant favorablement à l'importance de quelques mécanismes énonciatifs pour l'analyse de ce genre textuel nous nous bornerons à décrire quelques marques formelles énonciatives qui attestent le rôle joué par l'agent producteur dans ce genre : actant ou acteur ce qui nous amènera a une réflexion sur la possibilité de croiser quelques aspects théoriques des deux approches. Pour ce faire, la complémentarité des deux cadres nous fournira des outils théoriques importants pour l'analyse empirique des mécanismes énonciatifs dans les documents choisis.

3 Les données sur lesquelles se fonde ce travail sont issues d'enregistrements audio-visuels effectués en décembre 2009 dans cinq classes, de première année de deux universités portugaises (une publique et l'autre privée dans des facultés différentes). Il s'agit d'interactions verbales en salle de classe entre la chercheuse et les étudiants sur le thème entrepreneuriat. À partir de deux annonces publicitaires présentées, les élèves devraient exposer leur point de vue sur les rapports à être établis entre les documents présentés et la notion d'entrepreneuriat ${ }^{2}$.

\section{Genres textuels et aspects énonciatifs}

4 Comme dans cet article notre but est d'analyser les rapports qui se tissent entre les aspects génériques et les procédés énonciatifs dans les textes oraux développés en salle de classe, sur un thème bien défini, quelques aspects de l'approche interactionniste sociodiscursive $e^{3}$ nous paraissent particulièrement intéressants (Bronckart, 1997). D'une part, l'évidence que lors de la production d'un texte empirique déterminé, l'agent producteur doit adapter/adopter des modèles de texte présents dans sa mémoire à long terme. Ces ensembles de textes/genres constituent des modèles à partir desquels toute production textuelle nouvelle devra nécessairement s'inspirer. En plus, tout texte (qui appartient à un genre textuel $\left.{ }^{4}\right)$ est une unité communicative globale située dans une pratique sociale. De cette façon, les procédés sémiotiques qui y sont utilisés (mécanismes énonciatifs, par exemple) sont contraints par le genre/l'activité sociale où le texte s'intègre. D'autre part, l'importance de la méthode descendante d'analyse, comme le préconise cette perspective: l'analyse de la matérialisation sémiotique des textes doit être faite de l'activité/l'agir ${ }^{5}$ aux textes et puis aux ressources linguistiques y présentes (Bronckart, 2004, 113-123). En définitive, il semble donc que les textes produits par les actants dans des contextes sociohistoriques particuliers relèvent des pratiques sociales déterminées - médiatisées par des genres textuels divers - et des types d'agir différents. Et dans ce travail nous attirons notre attention sur un type d'agir particulier - l'agir des élèves en salle de classe lorsqu'ils 
produisent un débat d'idées sur un thème déterminé (l'entrepreneuriat dans notre cas). À ce propos, le débat d'idées est considéré un genre textuel et, par conséquent, nous pouvons identifier, dans les documents qui seront analysés, quelques aspects récurrents, prototypiques du genre textuel analysé, bien qu'il y en ait d'autres qui peuvent varier selon des aspects contextuels divers.

5 Au plan théorique, deux dimensions de cette approche nous semblent intéressantes. D'abord, elle cherche à articuler d'une façon dynamique la situation d'action langagière ${ }^{6}$ en cours aux propriétés sémiotiques des textes. Puis, cette même approche considère les aspects psychologiques et sociaux des situations de production des textes à partir de deux ensembles de représentations: les représentations portant sur le contexte de production (celles mobilisées à propos de l'espace-temps, des instances d'agentivité et des buts de l'interaction) et sur les contenus thématiques mobilisés lors de la production des textes.

6 Tout en considérant que les textes ont un triple statut: psychologique, linguistique et praxéologique, la question qui se posera dès lors est de déterminer comment les propriétés énonciatives des textes qui sont mobilisées dans l'interaction par les actants (tous les individus impliqués dans l'agir, comme nous l'avons souligné précédemment) peuvent être mises en lien avec les ressources génériques. Plus spécifiquement, la question qui nous intéressera en particulier sera de déterminer si les actants impliqués dans ce type d'agir se présentent linguistiquement comme des agents ou des acteurs de leur agir.

\section{Le genre débat d'idées - caractéristiques générales}

7 Pour la majorité des gens, le genre débat d'idées est propre à la télévision ${ }^{7}$ (au moins, c'est le modèle prototypique que nous avons tous). Les gens sont invités à intervenir sur le plateau (experts, personnalités du monde social et économique ou des personnes anonymes) et leur parole est influée par le dispositif médiatique qui les entoure : le choix des mots utilisés par les invités pour répondre aux questions de l'interviewer ou de l'animateur, la façon dont ils sont montrés. En effet, le débat doit être le plus spectaculaire possible, comme ce dispositif l'impose. Ainsi, c'est cette espèce de modèle qui nous permet d'avoir une représentation de ce que c'est un débat.

8 Cependant, comme l'affirment Dolz, Schneuwly \& Pietro $(2010,215)$, ce n'est pas exactement ce modèle qui doit être travaillé en salle de classe. Pour ces auteurs, il y a trois types de débats qui peuvent être enseignés: le débat d'opinions ayant pour caractéristique la controverse, le débat délibératif et le débat pour la résolution de problèmes et. Ils ont tous un trait commun : l'argumentation y est toujours associée.

9 Le premier a trait à la présentation des opinions, des croyances à propos d'un sujet. Son but est d'influencer/de transformer la position d'un interlocuteur (ou même celle de l'agent producteur) sur un thème donné. Par des confrontations et des déplacements de sens, le débat ici est un moyen efficace de mieux comprendre un sujet polémique et de découvrir plusieurs opinions sur le même objet.

Par le deuxième, les connaissances et les savoirs sont discutés en groupe et présentés par chaque élève. La solution du problème sera collective et sera atteinte à partir des contributions individuelles.

11 Par rapport au troisième, nous remarquons que le débat correspond à l'explicitation des points de vue de chaque individu sur un thème proposé. Dans ce type de débat, l'objectif 
de l'argumentation est présenter une/plusieurs prise(s) de position sur un certain sujet. Et dans cette contribution, c'est le débat pour la résolution de problèmes qui nous retiendra plus particulièrement. Comme nous l'avons mentionné, auparavant, les données ont été obtenues à partir d'un débat en salle de classe sur le thème entrepreneuriat.

\section{Le débat pour la résolution de problèmes : analyse générique et agentivité}

Dans ce qui suit, nous aborderons, tout d'abord, les aspects contextuels des textes analysés et plus particulièrement les paramètres du genre textuel dans l'agir concerné (agir des élèves en salle de classe). Ensuite, notre travail opère une centration forte sur la description des ressources énonciatives choisies dans ces documents. L'intérêt porté sur cette description renvoie à l'un de nos axes de réflexion : le rôle attribué à l'actant dans ces textes. Serait-il un agent ou un acteur dans cette configuration de l'agir?

\section{Contextualisation}

On peut affirmer qu'en gros le débat implique toujours au moins deux interlocuteurs qui négocient et reformulent leurs idées sur un sujet quelconque. C'est pourquoi ce genre textuel est un instrument intéressant pour travailler les capacités argumentatives des élèves lorsqu'ils soutiennent des points de vue. Et ce processus de choix ou de découverte implique des dimensions linguistiques, sociales, cognitives. Ces traits saillants sont en rapport direct avec le contexte de production de ce genre textuel.

Du point de vue des contextes physique et socio-subjectif, les débats analysés et transcrits ${ }^{8}$ ont été réalisés dans des salles de classes des institutions publiques et privées, pendant le mois de Décembre 2009. D'un côté, il y avait une chercheuse (étrangère pour les élèves dans ce contexte) ; et de l'autre, les élèves. Le professeur avait déjà expliqué auparavant ce qui allait se passer en salle de classe : il y aurait l'intervention de quelqu'un qui voulait obtenir des données pour sa recherche en cours. Alors, l'objectif de ce débat n'avait aucun rapport avec le contenu donné en classe. Le but était de fournir des informations pour le chercheur. On peut donc aller jusqu'à dire que cette portée plutôt imposée par le professeur aux élèves pourrait avoir des conséquences sur la matérialisation textuelle analysée (surtout sur les procédés énonciatifs choisis), comme nous allons constater par les textes.

\section{Instances d'agentivité - analyse des ressources linguistiques}

Dans le corpus analysé, nous avons choisi quelques constructions linguistiques qui nous permettent de nous interroger sur le rôle des traces d'agentivité qui y sont présentes. Et nous y identifions comme très fréquents quelques constructions linguistiques de subordination avec les verbes achar (trouver), pensar (penser) et crer (croire) à la première personne, qui introduisent un contenu propositionnel (un complément prédicatif). Ce sont des constructions du type $(E u)$ acho/penso/ creio que $X$, qu'on exemplifie ici à travers les énoncés de 1 a 6 :

(1) Acho que é o mesmo produto ( $\left.\mathrm{v}^{9} .2\right)$

(2) Enquanto da Ta : a da TAP. são produtos novos. E são outras formas três formas

de desconto e é uma coisa nova... até parece que para : â : se calhar responder de 
outra forma a necessidade â : dos consumidores. E daí eu acho que o anúncio da TAP seja mais empreendedor (v. 2)

(3) Penso que empreendedorismo basicamente é isso, é agarrar-me em algo (v. 1)

(4) Penso que daí [o anúncio da TAP] seja mais empreendedor, que o Banco Alimentar (v. 2)

(5) Eu creio que existe mais empreendedorismo no plano da parte da TAP (v. 1)

(6) Não creio que só exista um empreendedorismo na maneira como se passa uma mensagem (v. 1) modalité épistémique, plus spécifiquement une validation partiale de la relation prédicative. Au niveau du complément, le mode du verbe est soit à l'indicatif (énoncés 1, 3 et 5), soit au «conjuntivo" (le subjonctif, en français) (énoncés 2,4 et 6). Le «conjuntivo" marque la construction d'une situation d'énonciation décrochée par rapport à la situation d'énonciation en cours, ce qui correspond sémantiquement à l'affaiblissement du degré de validation de la relation prédicative par le sujet de l'énonciation.

Mais il y a d'autres constructions qui introduisent un contenu propositionnel et qui constituent des marqueurs de modalité épistémique. C'est le cas des expressions qui introduisent un point de vue, construites toujours par rapport au sujet énonciateur. Dans les énoncés suivants, nous présentons quelques exemples des formes pronominales de la première personne mim et minha:

(1) Para mim, o empreendedorismo é agarrar uma ideia muito simples e transformá-la num caso de sucesso (v. 1)

(2) O Banco Alimentar é um pedido para se contribuir para pessoas com necessidades. [...] Esta é a minha posição, a minha maneira de ver. (v. 1).

Nous pouvons constater que ces constructions mettent en évidence trois types de faits linguistiques employés très fréquemment dans ce corpus de textes du genre débat d'idées:

I. la subordination

II. les constructions prédicatives, surtout avec les verbes pensar, crer, achar

III. la première personne

En résumé, ces données linguistiques suggèrent la question générale du rapport entre l'hétérogénéité énonciative et la validation subjective, qui sera l'objet de cette contribution, en réfléchissant sur l'hypothèse d'une fondamentation du concept d'actant l'entité impliquée dans l'agir -, qui joue un rôle important dans ce genre textuel.

\section{La subordination - quelle caractérisation énonciative?}

Dans une perspective de la construction de la référence, il est nécessaire de considérer les paramètres métalinguistiques, qui permettent l'ancrage situationnel des valeurs dont toutes formes linguistiques de l'énoncé - et pas seulement le verbe introducteur - sont marqueurs. Le processus de subordination correspond à un marqueur complexe d'une opération de détermination considérée à deux niveaux : à celui de la construction de la relation prédicative associée à la proposition principale et à celui de la détermination énonciative de la relation prédicative complexe, en marquant la détermination des valeurs temporelles, aspectuelles et modales de l'énoncé (Deschamps, 1997).

21 Par conséquent, pour une approche énonciative de la subordination (cela veut dire, en envisageant un modèle énonciatif de la subordination), c'est fondamental de nous situer dans une perspective "transcatégoriale ", et de considérer la subordination comme un 
processus à travers lequel le syntaxique et le sémantique sont décrits dans une même séquence d'opérations. À partir de cela, pour une convenance méthodologique et pour une explication métalinguistique, on intègre les divers niveaux de la construction de l'énoncé dans un « tout » descriptif et sans discontinuité.

Pour l'étude de la subordination, Culioli (1982) signale une orientation générale dans le cadre de la TOPE, en affirmant qu' « une lexis peut être composée avec une autre lexis et [...] on peut construire une relation de repérage entre lexis » (1982 [aussi 1999a, 101]).

Ce présupposé théorique nous permet de surpasser le concept traditionnel de la subordination comme forme d'association entre deux structures à travers un processus d'imbrication, ce qui provoque, dans le cas de la subordination nominale, un rapport de dépendance entre les deux structures ou propositions. Cela nous permet, donc, de redéfinir cette construction comme une relation prédicative complexe dont la relation " embriquée ", en instanciant le lieu d'un argument $C_{1}$ dans la relation «imbricante » (Deschamps, 1997), est introduite par que, « l'image du premier énonciateur » (Culioli, 74, 12) qui représente, donc, «l'acte assertif du $S_{0}$, origine de toute énonciation » (Ibid,12). D'après Wyld (2001), on est devant « une conception du mécanisme d'enchâssement selon laquelle la structure enchâssée [...] est posée comme venant saturer une placecomposante de la structure enchâssante [...] » (Ibid., 9).

En conséquence, les questions présentées s'orientent dans le sens d'une possibilité de description et d'explication du processus de subordination différent des autres propos, parce que, avant tout, on problématise la centralité du verbe introducteur dans l'établissement de ce type de relation. Donc, une fois que cette proposition se situe dans le cadre de la TOPE, elle provoque nécessairement des conséquences d'ordre simultanément terminologique et conceptuelle en ce qui concerne la construction du concept d'imbrication. La subordination est conçue comme un marqueur de la localisation du complément (la relation «embriquée ») par l'énonciateur. Mais cette localisation par rapport à un système référentiel pose le problème de la dissociation entre la source énonciative $\left(\mathrm{S}_{0}\right)$ et la source modale, cela veut dire, de ce que dans ce travail nous interprétons comme un indice d'actoralité.

\section{Des constructions prédicatives avec les verbes pensar, crer, achar}

En présence de la complexité associée à la détermination modale des énoncés, la question qui se pose est : comment se construisent les valeurs modales dans les énoncés complexes dont les relations prédicatives, liées par une "relation d'imbrication» (qui est une relation de localisation), peuvent présenter de différentes valeurs modales, ou, au moins, de différentes sources modales?

Cela nous amène encore à nous interroger sur le rôle des verbes - pensar, julgar, crer, achar , supor - comme des formes qui marquent une valeur modale épistémique du domaine du non-certain. On le fait en assumant que, comme le propose Culioli, le centrage du travail de calcul des valeurs modales sur le paramètre abstrait subjectif (S) du système référentiel (Sit) correspond à un moyen théorique de décrire le fonctionnement des ces verbes, notamment les valeurs modales susceptibles d'être véhiculées au niveau de la relation « imbriquée».

27 Ainsi, on constate qu'une variation au niveau du sujet de l'énoncé (l'argument $\mathrm{C}_{0}$ de la relation imbricante) implique qu'il y ait la construction de différentes valeurs modales. Ce 
fait pose le problème de la nature de la source énonciative et de sa définition métalinguistique au sein des sites construits à partir de $\mathrm{Sit}_{0}$ en mettant en évidence le besoin de considérer aussi, pour la description métalinguistique, d'autres localisateurs situationnels dérivés.

Cette possibilité est mentionnée par Culioli. Après la présentation du système complexe des coordonnés énonciatives $\left(\mathrm{Sit}_{0}, \mathrm{Sit}_{1}, \mathrm{Sit}_{2}\right)$, cet auteur affirme «[...] Ce système est minimal et peut être enrichi de façon réglée par la construction d'autres repères " (Culioli, 1982, 17). Par ailleurs, il renforce la nature simultanément " absolu et ajustable " de l'origine énonciative $\mathrm{Sit}_{0}$ et exemplifie les procès de construction d'autres origines énonciatives, dérivées de $\mathrm{Sit}_{0}$ :

[...] cette origine est à la fois absolue et ajustable. Mais à partir de cette origine absolue, on va construire (1) une origine de locution (2) des origines dérivées de l'origine absolue soit par translation (d'où une origine translatée, qui conserve les propriétés de l'origine absolue) soit par rupture (origine décrochée). (Ibid., 17)

La première peut être identifiée, par exemple, dans l'imparfait des langues romanes ; la seconde se construit, chaque fois que l'on engendre une origine fictive, détachée de l'actualité, d'où l'on envisage les successions d'événements (états; transitions). (Ibid., 17)

Ainsi, pour ne citer que quelques cas bien connus [...], dans les indications scéniques, dans une notice biographique, dans les reportages ou les récits historiques. Mais aussi dans des schémas modaux, où l'on envisage des chemins possibles ou nécessaires, ou encore dans les énoncés performatifs, grâce auxquels on ne décrit pas, mais on accomplit par le fait même de dire. (Ibid., 167) ${ }^{10}$

Pour la description métalinguistique, c'est, donc, à partir du domaine de la modalité épistémique que nous pouvons identifier d'autres localisateurs dérivés.

Le problème de la source énonciative et de sa définition métalinguistique a été l'objet de plusieurs propositions. Basé sur cette perméabilité du système référentiel à l'intégration des origines dérivées, Wyld, par exemple, propose un localisateur supplémentaire, susceptible d'enrichir le système complexe des coordonnées énonciatives : un « repèreorigine dérivé », avec la notation métalinguistique $\operatorname{Sit}_{0 \mathrm{~d}}\left(\mathrm{~T}_{0 \mathrm{~d}}, \mathrm{~S}_{0 \mathrm{~d}}\right)$, qui correspond à un « repère supplémentaire éventuel », ou à "une sorte de copie déplacée » (Wyld, 2001, 17-19). Dérivé de $\mathrm{Sit}_{0}$, ce localisateur conserve, sous des degrés variables, les propriétés de l'origine absolue ce qui - comme l'affirme Wyld - viabilise la conception de diverses soustypes d'origines dérivées et permet, de cette façon, l'avantage sur la dichotomie culiolienne entre l'« origine translatée » et l'« origine fictive » ou l'« origine décrochée » (Culioli, 1993, 167).

31 Campos considère ce problème général de la dissociation énonciative en proposant deux types distincts de disjonction énonciative par rapport à Sit ${ }_{0}$ : une disjonction abstraite et une disjonction référentielle. D'une part, elle considère une "origine énonciative abstraitement [et pas référentiellement] disjointe de $\mathrm{Sit}_{0}$ " - (Campos, 2001, 170) - qui peut être marquée linguistiquement par la première personne grammaticale. Ceci tient, de notre point de vue, à la description du sujet comme un «indicateur de subjectivité", comme l'a clairement montré Benveniste (1966). D'autre part, Campos note la présence d'une «origine énonciative référentiellement distincte de $\mathrm{Sit}_{0}$ " (Ibid., 170), qui correspond à la situation de locution $\mathrm{Sit}_{1}$, incluant le sujet modal $\mathrm{S}_{1}$, et qui peut être marquée linguistiquement par la troisième personne grammaticale, correspondante, donc, à un marqueur d'un calcul référentiel. 

subordination. Notamment, la proposition de Campos se présente plus adéquate opératoirement pour désigner le support modal marqué par de différentes personnes grammaticales. s'impose. La question qu'on peut se poser à partir de la réflexion de Benveniste est la suivante : comment définir une valeur de subjectivité construite de façon particulière, dans le domaine de la modalité épistémique?

Le sujet énonciateur, loin de nous perdre dans les sables mouvants de la subjectivité, devient [...] le pôle de stabilité incessamment renouvelé, à partir duquel le langage va pouvoir acquérir la variabilité nécessaire à son fonctionnement (Robert, 1994, 228)

Selon Culioli, le sujet énonciateur, comme paramètre énonciatif et source de la détermination de l'énoncé, est «[...] le premier sujet énonciateur, qui nous fournit l'origine de l'espace intersubjectif de toute situation énonciation $»^{11}$. On peut constater à partir de cette affirmation que le sujet n'est pas identifié comme la source d'une subjectivité stricte, puisque l'énonciation se règle plutôt par l'intersubjectivité que par la subjectivité. Pourtant, lorsqu'on a, comme dans les énoncés de 1 à 8 , la construction d'une source modale abstraitement disjointe de $\mathrm{S}_{0}$, cela veut dire, marquée linguistiquement par la première personne, ce sujet modal, en préservant les propriétés de la source énonciative, ne correspond pas à la construction d'une nouvelle origine de locution référentiellement distincte. Par contre, il correspond à la « subjectivation » de $\mathrm{S}_{0}$, comme source d'un processus d'inférence, à partir de l'interprétation de quelques indices.

Ainsi, sa description métalinguistique comme agent cognitif semble exiger (on l'a déjà suggéré) le recours au concept de subjectivité. L'assomption partiale, quel que soit le degré de validation de la relation prédicative, se construit par un dédoublage de l'origine énonciative qui, dans ce cas, est construite aussi comme un agent de construction et de transmission de connaissance, c'est-à-dire, comme la source d'une subjectivité ou comme le responsable d'une validation subjective.

Cette réflexion nous fait avancer vers le troisième fait linguistique commun aux énoncés de 1 à 8 : la première personne.

\section{La première personne comme « indice de subjectivité »}

La première personne explicite, pas seulement l'instance de l'énoncé $\left(\mathrm{S}_{2}\right)$, mais aussi l'instance de l'énonciation $\left(\mathrm{S}_{0}\right)$. Par conséquent, comme forme dont dispose l'énonciateur pour s'instituer comme support de la prédication, le «je » signale une localisation de l'identification entre $\mathrm{S}_{2}$ et $\mathrm{S}_{0}$.

Une référence à cette coïncidence entre l'instance de l'énoncé et l'instance de l'énonciation - selon quelques auteurs «fonction prédicative» et "fonction référentielle » (voir, par exemple, Joly, 1994; O’Kelly, 1996) - a été déjà présente dans un texte du grammairien latin Priscian (vi ${ }^{\mathrm{e}}$ siècle), lorsque, après la tradition du philosophe de l'antiquité Apollonios Dyscole, il affirme que « La première personne, c'est quand celle qui parle, parle d'elle-même [...] $\aleph^{12}$. Contrairement au grammairien alexandrin Dionysius Thrax (siècle I), qui classifie la personne en fonction de son rôle alocutoire, Apollonios 
Dyscole explicite le fait de la première personne marquer, par le moyen de l'énonciation, la construction de son « auto-proclamation » comme sujet de l'énoncé.

Il y a une divergence de ce type entre Benveniste et Guillaume, quand on compare les réflexions que les deux auteurs proposent sur la personne, particulièrement sur les pronoms. D'une part, dans la continuité de Dionysius Thrax, Benveniste considère uniquement la dimension alocutoire des formes personnelles et c'est cela qui l'amène à classifier la troisième personne comme « non-personne » :

Dans les deux premières personnes, il y a à la fois une personne impliquée et un discours sur cette personne. "Je" désigne celui qui parle et implique en même temps un énoncé sur le compte de «je»: disant «je», je ne puis ne pas parler de moi. À la $2^{e}$ personne, le «tu » est nécessairement désigné par « je » et ne peut être pensé hors d'une situation posée à partir de "je »; et, en même temps, «je » énonce quelque chose comme prédicat de «tu ». Mais de la $3^{\mathrm{e}}$ personne, un prédicat est bien énoncé, seulement hors du «je-tu»; cette forme est ainsi exceptée de la relation par laquelle «je » et «tu » se spécifient. Dès lors, la légitimité de cette forme comme 'personne' se trouve mise en question. [...]. (Benveniste, 1966, 228).

D'autre part, Guillaume continue à reconnaître le statut particulier des personnes interlocutoires (première et troisième personnes), mais il attribue une "fonction prédicative » à la catégorie de personne en général. Il conçoit que la première personne se définit dans une relation avec le « hors de soi » et, donc, avec la troisième personne, qu'il considère qui est sous-jacente à toutes les personnes. Conséquemment, cet auteur considère que la troisième personne est le fondement du système linguistique :

[...] notre conception grammaticale de la personne comporte, partout et toujours, une certaine évasion grammaticale de la personne parlante. Cette évasion existe, sans qu'on en fasse l'observation, en toutes les personnes, y compris la première. Car qu'est-ce que la première personne : c'est la personne qui parle, parlant d'ellemême, c'est-à-dire une personne qui est première, en ce qu'elle parle, et troisième en ce qu'il est parlé d'elle par elle-même. Le je est quelque chose de très compliqué. On sait que sa complication étymologique est, elle aussi, grande. Ego représente un assemblage de pronoms [...]. (Guillaume, ([1947-48] 1988, 183).

41 L'importance attribuée par Guillaume à la «fonction prédicative » de la personne est basée sur le fait de que la nature symétrique des personnes interlocutoires («[...] entre lesquelles, comme entre deux pôles, le langage s'inscrit et se développe » (id, 183) n'est pas un trait universel mais un trait particulier des langues indo-européennes.

Aussi Milner, parmi d'autres auteurs, affirme le besoin de distinguer le sujet d'énonciation - "[...] un concept positif de la linguistique, qui, a des fins de pure description » (Milner, 1978, 119) - du sujet de l'énoncé et montre que par l'identification de ces deux instances se produit une subjectivation de l'énoncé. Cet auteur affirme :

[...] pour penser la possibilité du je, il lui faut poser tout énoncé peut être rapporté à un point, dont on ne suppose rien sinon qu'il énonce. Mais immédiatement, un tel concept s'ouvre à une autre lecture : le point auquel l'énoncé est rapporté est en même temps posé comme un sujet, et la possibilité demeure qu'il subjective l'énoncé d'une manière qui échappe à la représentation. (Ibid., 119-120)

43 Ce rappel de certaines réflexions grammaticales nous permet de systématiser notre proposition de la façon suivante. En intégrant les «formules modales» (eu) penso, (eu) julgo, (eu) creio, (eu) acho, (eu) suponho, la première personne ne marque pas seulement une localisation d'identification entre $S_{2}$ et $S_{0}$, mais aussi, et à cause de la valeur modale épistémique du domaine du non-certain, la construction d'une source énonciative, 
abstraitement disjointe de $\mathrm{S}_{0}$, responsable de la validation partielle d'un contenu propositionnel. chez Benveniste (1966), une référence à la spécificité de l'emploi du pronom personnel de la $1^{\text {re }}$ personne, comme «indicateur de subjectivité ». On dirait, donc, que la première personne grammaticale ne correspond à un indice de subjectivité qu'en cooccurrence avec certains prédicats, notamment avec les prédicats qui intègrent des verbes qui permettent l'expression d'une opinion basée sur un point de vue. Selon Franckel et Lebaud, ces prédicats constituent « la catégorie des prédicats subjectifs", une fois qu'ils :

[...] impliquent une identification entre sujet du prédicat et sujet de l'énonciation, telle que toute dissociation conduit à des relations d'inférences dont les conséquences modales sont variables et plus ou moins nettement repérables [...] Si un prédicat non-subjectif «se prête à une assertion de l'énonciateur à toutes les personnes [...]», un prédicat subjectif registre «[...] une dissymétrie entre les emplois à la première et à la deuxième personne [...]». (Franckel et Lebaud, 1990, 52).

Cette réflexion autour de ces trois faits linguistiques présents dans ce corpus d'analyse - la subordination comme validation subjective, la $1^{\text {re }}$ personne comme indice de subjectivité et le fonctionnement particulier des prédicats subjectifs - nous amène aussi à un possible rapprochement au performatif austinien. Campos, en cherchant de définir le concept de performativité par une description métalinguistique (pour dépasser la faute des critères linguistiques qui soutiennent la définition de Austin), affirme que les performatifs se caractérisent par "l'identification entre $\mathrm{Sit}_{2}$ et $\mathrm{Sit}_{0}$ " $(1998,35)$, puisque les deux événements, énonciatif et phénoménal, sont coïncidents. Cela veut dire que ces deux événements de nature distincte sont validés dans le même intervalle d'instants, opération qui est marquée par le présent grammatical, d'emploi pratiquement exclusif dans ce corpus. Cela nous permet de nous approcher légèrement du performatif austinien et de parler de « pseudo-performativité».

Ce qui apparait clairement, c'est la simultanéité entre une occurrence phénoménale et une occurrence linguistique qui, en décrivant la première, la construit linguistiquement. Alors, le dédoublage de l'origine énonciative correspond à une « mise-en-scène », parmi laquelle l'énonciateur s'instaure comme un sujet cognitif, garant de da (non)validation de la relation prédicative, le seul responsable du jugement produit ce qui, en soi-même, affaiblit le pouvoir assertif du jugement.

\section{En guise de conclusion}

Parmi les traits linguistiques des textes analysés, il reste à signaler l'importance des structures de subordination avec des prédicats subjectifs à la première personne qui marquent une valeur modale épistémique (du non-certain). Le choix de ces formes contribue à la description des sujets (et de leur agir) dans cet univers textuel.

L'acte de discuter un sujet déterminé (débat résolution de problèmes) constitue une action directement produite par un actant (ici l'élève), mais le choix de certaines constructions linguistiques semble éloigner ce même actant de ce qu'il dit/il affirme. Par l'analyse des paramètres linguistiques pris en considération, il faut souligner l'importance réduite de cet élève du point de vue de la relation élève - chercheur - collègues. Il se donne à voir comme quelqu'un qui n'a pas l'un des premiers traits qui distingue l'entrepreneur de toute 
autre personne à l'œuvre dans les organisations : sa propension à bien définir ses projets, puis à passer à l'action pour les réaliser. On peut faire remarquer que, par ses productions langagières, dans son plein exercice praxéologique, d'un agir déterminé, l'élève se présente comme un pseudo-acteur. Un acteur qui ne prend aucune position claire sur un sujet proposé en classe.

Cette réalité attestée par les exemples nous incite à revenir sur les aspects contextuels de production de ces textes. Ceux-ci ont été produits en salle de classe visant à atteindre le but d'une recherche particulière (expliqué par le professeur de ces mêmes classes). Cette instance joue un rôle hiérarchique important dans l'institution et peut avoir une influence sur le choix des formes linguistiques utilisées par les élèves dans des pratiques spécifiques. À ce propos, on peut supposer que le contexte socio-subjectif de cet agir a une incidence forte sur ce que chacun juge important à dire et sur le choix des formes linguistiques utilisées.

Certes, ces quelques exemples ne prétendent évidemment pas à l'exhaustivité et ne constituent que quelques balises intéressantes pour l'étude du rôle des instances agentives dans un agir déterminé à partir des procédés linguistiques utilisés textuellement. D'autres travaux futurs portant sur un ensemble plus diversifié de données empiriques peuvent attester l'importance des aspects contextuels sur le rôle attribué aux instances agentives dans d'autres types d'agir.

Pour conclure, en dépit des spécificités des deux cadres théoriques utilisés : d'une part le ISD, pour l'analyse des textes au niveau des aspects génériques ; d'autre part la TOPE pour la description des formes et des constructions linguistiques comme des marqueurs d'opérations et des valeurs énonciatives, nous avons pu constater que la complémentarité des deux approches a largement contribué à l'identification du rôle des instances énonciatives dans l'agir des élèves dans ce genre textuel spécifique.

\section{BIBLIOGRAPHIE}

BENVENISTE, Émile, Problèmes de linguistique générale 1, Paris, Gallimard, 1966.

BENVENISTE, Émile, Problèmes de linguistique générale 2, Paris, Gallimard, 1974.

BRONCKART, Jean-Paul, « Le travail comme agir et la formation pour l'analyse du travail », in Bronckart, Jean Paul et le groupe LAF (éd.), Agir et discours en situation de travail, Cahiers de la section des sciences de l'éducation 103, 2004, p. 87-100.

BRONCKART, Jean-Paul, « S'entendre pour agir et agir pour s'entendre », in Baudouin, J.-M. ; Friedrich, J. (éds.), Théories de l'action et éducation, Bruxelles, de Boeck \& Larcier, 2001, 133-154.

CAMPos, Maria Henriqueta Costa, "Enunciação mediatizada e operações cognitivas", in Da Silva, A. S. (org.) Linguagem e cognição. A Perspectiva da linguística cognitiva, Braga, Associação Portuguesa de Linguística, Universidade Católica Portuguesa - Faculdade de Filosofia, 2001, 325-340.

CULIOLI, Antoine, Pour une linguistique de l'énonciation. Opérations et représentations 2, Paris, Éditions Ophrys, 1999a. 
CULIOLI, Antoine, Pour une linguistique de l'énonciation. Opérations et représentations 3, Paris, Éditions Ophrys, $1999 \mathrm{~b}$.

CULIOLI, Antoine, «Les modalités d'expression de la temporalité sont-elles révélatrices de spécificités culturelles ?» Interfaces 5, CRDP, Paris, 1993 (aussi 1999a, 159-178).

CULIOLI, Antoine, « Rôle des représentations métalinguistiques en syntaxe », Université Paris VII / DRL, Coll. ERA 642, 1982 (aussi 1999a, 95-113).

CULIOLI, Antoine, « À propos des énoncés exclamatifs », Langue Française 22 : 6-15, 1974 (aussi 1999b, 113-123).

DESCHAMPS, Alain, « Traitement énonciatif des paramètres des compléments propositionnels des verbes ", in Rivière, C. ; Groussier, M.-L. (eds.) La notion, Paris, Éditions Ophrys, 1997, p. 60-74. DOLZ, Joaquim, SCHNEUWLY, Bernard et DE PIETRO, Jean-François, « Relato da Elaboração de uma sequência. O debate público » (trad. De Roxane Rojo), in Pour un enseignement de l'oral : Initiation aux genres formels à l'école, Paris, ESF Editeur, 1998, p. 213-239.

DUCROT, Oswald, Le Dire et le Dit, Paris, Minuit, 1984.

FRANCKEL, Jean-Jacques et LEBAUD Daniel, Les figures du sujet. À propos des verbes de perception, sentiments, connaissance, Paris, Éditions Ophrys, 1990.

GUILLAUME, Gustave, Leçons de linguistique, série C, vol. 8, Québec, Presses de l'Université Laval, Lille, Presses Universitaires de Lille [1947-48] 1988.

JOLY, André, « Pour une théorie générale de la personne », Faits de Langues 3, 1994, p. 45-54.

O'KELLY, Dairine, «A la Recherche d'une valeur fondamentale : le cas de la personne », Modèles Linguistiques 33 T. XVII, Fasc. 1, 1996, p. 177-185.

KERBRAT-ORECCHIONI, Catherine, L'Énonciation. De la subjectivité dans le langage, Paris, Armand Colin, 1980.

MAINGUENEAU, Dominique, Les termes clés de l'analyse du discours, Paris, Seuil, 1996.

MILNER, Jean-Claude, L'amour de la langue, Paris, Éditions du Seuil, 1978.

PINTO, Rosalice, « Gêneros textuais em atividades sociais : uma abordagem epistêmico-linguísticopraxiológica ", in Anais do VI Congresso Internacional da AbRALIN v.1. João Pessoa, Ideia (CD-Rom publ.) vol. 1, 2009, p. 2461-2469.

PINTO, Rosalice et VALENTIM, Helena T., "Uma descrição linguístico-textual. Que interfaces teóricas possíveis ? » in Werneck dos Santos L. et al. (orgs.), Anais do XV Congresso da Assel-Rio. Linguagens em diálogo : Pesquisa e ensino na área de Letras. Faculdade de Letras da UFRJ. Publ. em CD-rom, 2009.

PINTO, Rosalice et TEIXEIRA, Carla, « A agentividade no Agir Publicitário : uma análise multimodal, IV » Seminário Internacional de Linguística da Cruzeiro do Sul (IV SIL), Actas do IV Simpósio Internacional de Linguística, São Paulo, Universidade Cruzeiro do Sul, 2011, p. 203-215.

ROBERT, Stéphane, «Sur le rôle du sujet énonciateur dans la construction du sens : liens entre temps, aspect et modalité ", in Yaguello, M. (ed.) Subjecthood and subjectivity, Paris, Éditions Ophrys, 1994, p. 209-230.

VALENTIM, Helena T., Um estudo semântico-enunciativo de predicados subjectivos do português. Tese de Doutoramento em Linguística, especialidade em Semântica. FCSH-UNL (non publiée), 2005.

VALENTIM, Helena T. et PINTO, Rosalice, « Marcas Actanciais em inquéritos por questionário : uma abordagem linguístico-textual », in Sell, M. ; Guimarães, A.M.M. (orgs), Anais do Congresso 
Internacional Linguagem e Interação II. São Leopoldo (Rio Grande do Sul) : Casa de Leiria ISSN

2177-5702 (CD ROM, Revista Caleidoscópio), 2009.

voloschinov, Valentin Nikolaevich, Le Marxime et la Philosophie du langage, trad. fr., Paris, Minuit, 1977.

WYLD, Henry, Subordination et énonciation, Cahiers de Recherche (numéro spécial), Paris, Éditions

Ophrys, 2001.

\section{NOTES}

1. Jean-Paul Bronckart établit des différences entre actant, acteur et agent. L'actant est la source d'un agir donné ; le terme acteur est utilisé lorsque les mises en forme érigent l'actant en une source de procès dotée de capacités, de motifs et d'intentions et le terme agent lorsque les mises en forme n'attribuent aucune de ces propriétés à la source d'un procès - Jean-Paul Bronckart, 2004. « Le travail comme agir et la formation pour l'analyse du travail », in Jean-Paul Bronckart et le groupe LAF, Agir et discours en situation de travail, Cahiers de la section des sciences de l'éducation 103, 2004, p. 111.

2. Nous remercions au Prof. Maria do Céu Caetano de l'Université Nova de Lisbonne qui nous a permis la réalisation du débat dans sa classe de linguistique à l'Université Nova de Lisbonne en décembre/2009.

3. Jean-Paul Bronckart, Activité langagière, textes et discours. Pour un interactionnisme sociodiscursif, Lausanne, Delachaux et Niestlé, 1997.

4. Un genre textuel ici correspond à une pratique socioculturelle historiquement située et subit des contraintes contextuelles diverses. C'est exactement notre connaissance sur les modèles de genres textuels qui nous permet une certaine économie cognitive lors de la production / interprétation d'un texte. N'oublions pas qu'un genre textuel a des caractéristiques prévisibles et il en a d'autres qui sont variables.

5. Selon l'ISD l'activité a un statut théorique ou interprétatif et implique les dimensions motivationnelles et intentionnelles mobilisées au niveau collectif. L'agir désigne toute forme d'intervention orientée d'un ou plusieurs humain(s) dans le monde. Comme cette contribution vise plutôt le niveau praxéologique du genre textuel débat d'idées, nous préférons le terme agir pour qualifier les données. Tout en considérant qu'il est influencé par des aspects psychologiques divers.

6. Selon la perspective du ISD une situation d'action langagière peut être définie par les connaissances du contexte (paramètres matériels et socio-subjectifs de l'interaction) et celles du contenu thématique qui se présente sous la forme des macrostructures sémantiques élaborées par l'agent-personne sur un domaine de référence donné. - Jean-Paul Bronckart «S'entendre pour agir et agir pour s'entendre ", in Baudouin, J.-M. \& Friedrich, J. (éds.), Théories de l'action et éducation, Bruxelles, de Boeck \& Larcier, 2001, p. 133-154.

7. Patrick Charaudeau, 2001, "La télévision et l'autre-étranger. Conditions d'une étude ", in Charaudeau, P., Lochard, G ; Soulages, J.-C.; Fernandes, M. et Croll, A. La télévision et la guerre. Déformation ou construction de la réalité, Bruxelles, De Boecke Université, 2001, p. 8-26.

8. Les conventions des transcriptions utilisées sont celles adoptées par Laurent Filliettaz dans ses travaux sur l'analyse des interactions orales - Laurent Filliettaz, «Interaction, cognition et identités situées: une analyse praxéologique des transactions de service", in Bulletin de Psychologie, 57(1), p. 61-64.

9. Les énoncés présentés sont identifiés par la lettre « v », correspondante à "vidéo », suivie du numéro du vidéo analysé, suivant l'ordre chronologique de l'enregistrement. Il importe de rappeler que nous avons travaillé avec cinq classes différentes. 
10. Nous avons utilisé les caractères en italique pour mettre en relief les aspects importants pour la compréhension de l'importance des schémas modaux dans notre travail.

11. Antoine Culioli, 1973, Sur quelques contradictions en linguistique, Communications, 20, 1973, p. 88.

12. Jean Lallot, La grammaire de Denys le Thrace, traduction annotée, Paris, Éditions du CNRS (apud Dairine O'Kelly "Autour de la notion de personne ", in Claude Rivière ; Marie-Line Groussier (eds.), La notion, Paris, Éditions Ophrys, 1997, p. 299).

INDEX

Mots-clés : théorie des opérations prédicatives et énonciatives, genre textuel, agentivité

AUTEURS

HELENA T. VALENTIM

ROSALICE PINTO 